\title{
Index of Cases
}

Abingdon School District v. Schempp, 374 U.S. 203 (I963), 22n

Acanfora v. Board of Education, 359 F. Supp. 843 (D. Md. 1973), 491 F. 2d 498 (4th Circ. I974), cert. den. 4I9 U.S. 836 (I975), I9n, 245-5 I

Adamson v. California, 332 U.S. 46 (I947), 2 I, 80, 98-IOI

Albemarle Paper Co. v. Moody, 422 U.S. 405 (1975), I $32 \mathrm{n}$

Ambach v. Norwick, 44I U.S. 68 (1979), 26n, 262n

American Public Transit Association v. Goldschmidt, 485 F. Supp. 8 I I (D.D.C I 980), I94n

Atlantis Community, Inc. et al. v. Adams, 453 F. Supp. 825 (D. Colo. 1978), $208 \mathrm{n}$

Aumiller v. University of Delaware, 434 F. Supp. 1273 (D. Del. I 977), I 9n, 248-49

Baker v. Owen, 395 F. Supp. 294 (M.D. N.C. 1975), I77n

Bakke v. Regents of the University of California, 553 P. 2 d I 52 (Cal. Sup. Ct. 1976)

See Regents of the University of California v. Bakke

Barron v. Baltimore, 7 Peters 243 ( 1833 ), 67-68, 99-10I

Bartley v. Kremens, 402 F. Supp. I039 (E.D. Pa. 1975), I66n

Bates v. Little Rock, 36I U.S. 516 (1958), 26n

Berg v. Claytor, 436 F. Supp. 76 (D. D.C. 1977), 591 F. 2 d 846 (D.C. Circ. 1978), 243n

Blackwell v. Issaquena County Board of Education, 363 F. 2 d 749 ( 5 th Circ. 1966), I74n, I75n

Board of Education v. Rowley, 50 U.S.L.W. 4925 (1982), 22 I-24

Boddie v. Connecticut, 40 I U.S. 37I (I97I), 27n

Bolling v. Sharpe, 347 U.S. 497 (I954), I I4-I 8, 202n

Bothman v. Warren B., cert. den., 445 U.S. 949, roo S.Ct. I 597 (1980), I 8n, 198

See also Phillip B., in re 


\section{Index of Cases}

Boxall v. Sequoia Union High School District, 464 F. Supp. I I०4 (N.D. Cal. 1979), 204n

Bradley v. Vance, 436 F. Supp. 134 (D. D.C. I 977)

See Vance v. Bradley

Bradwell v. Illinois, 83 U.S. I 30 (1873), 106-7, 198

Breed v. Jones, 42 I U.S. 519 (1975), I79n, 182

Brown v. Board of Education, 347 U.S. 483 (I954), 22, 80, 95, I I 3-I9, I 25, 203, 208, 228, 256, 260

Buck v. Bell, 274 U.S. 200 (1927), 197-200

Buckholz v. Leveille, I 94 N.W. 2 d 427 (Ct. App. Mich. 1971), I63n

Burnside v. Byars, 363 F. 2d 744 (5th Circ. 1966), 174n

Califano v. Goldfarb, 430 U.S. 199 (1977), I44

Califano v. Webster, 430 U.S. 3 I 3 (1977), 1 47-48

Carter v. Gallagher, 452 F. 2d 3 I 5 (8th Circ. I972), I $32 \mathrm{n}$

Cherry v. Mathews, 4I 9 F. Supp. 922 (D. D.C. I976), I93n

Civil Rights Cases, I09 U.S. 3 ( 1883 ), I09, I 4 I

Cleveland Board of Education v. LaFleur, 4I 4 U.S. 632 (I974), 2 I 3n, 250, $273 \mathrm{n}, 278$

Corfield v. Coryell, 6 Fed. Cases 546 (E.D. Pa. 1 823), 82, 98-99

Cotner v. Henry, 394 F. 2d 873 (6th Circ. 1968), I9n

Craig v. Boren, 429 U.S. I90 (I976), I I 9n, I 24-26, I 45-50, I 59, I85, 27 I

Crandall v. Nevada, 6 Wall. 35 ( 1868 ), 208n

Custody of a Minor Child (Chad Green), 379 N.E. 2d I053, 393 N.E. 2d 836 (Mass. Sup. Jud. Ct. I978, I979), I 9n, I68-72, 186, 270

Cuyahoga County Association for Retarded Children and Adults v. Essex, 4 I I F. Supp. 46 (N.D. Ohio), 204n, 206n

Dandridge v. Williams, 397 U.S. 47 I (I970), I $27 \mathrm{n}$

Davis v. Southeastern Community College, 424 F. Supp. I34I (E.D. N.C. I 976), 574 F. 2d I I 58 (4th Circ. I978), 2 I6-I 8

See also Southeastern Community College v. Davis

De Funis v. Odegaard, 4 I 6 U.S. 3 I 2 (1974), I 8n, I I 6, I 32-33

Dennis v. United States, 34 I U.S. 494 (I 95I), 240n, 276

Dew v. Halaby, 3 I 7 F. 2d 582 (D.C. Circ. 1963), 243n

Doe v. Commonwealth's Attorney for the City of Richmond, 403 F. Supp. I 199 (E.D. Va. I975), 425 U.S. 903 (I976), I 9n, 230n, 235-37, 249, 277

Donaldson v. O'Connor, 493 F. 2d 507 (5th Circ. 1974)

See O'Connor v. Donaldson

Dred Scot v. Sandford, 60 U.S. 691 (1857), 67, 70-73, 87

Edwards v. California, 3 I 4 U.S. I 60 (I94I), 208n

Eisenstadt v. Baird, 405 U.S. 438 (1972), 23 In, 234-37

Enslin v. North Carolina, 2 I 4 S.E. 2 d 318 (N.C. Ct. App. 1975), 425 U.S. 903 (1976), I9n, 230n, 236-37

See also Doe v. Commonwealth's Attorney for the City of Richmond

Fialkowski v. Shapp, 405 F. Supp. 946 (E.D. Pa. 1975), 204n 


\section{Index of Cases}

Foley v. Conellie, 434 U.S. 29I (1978), 26n, 262n

Franks v. Bowman Transportation Co., 424 U.S. 747 (I976), I $32 n$

Frontiero v. Richardson, 4I I U.S. 677 (I973), 26n, 29n, I 19-26, I 38, I4549, I 59-60, I 84, I 96, I 99, 2 I 3-I 5, 232, 25 I, 26I-62

Fullilove v. Klutznick, 448 U.S. 448, I00 S.Ct. 2758 (1980), I8n, 29n, I 3 on, I 34, 220

G. H., in interest of, 2 I 8 N.W. 2 d 44I (Sup. Ct. N.D. 1974), 29n, I 99

Gault, re, 407 P. 2 d 760 (Sup. Ct. Ariz. 1965), 38 I U.S. I (1967), I 9n, I $55 \mathrm{n}, \mathrm{I} 64, \mathrm{I} 80-84,200-201$

Gay Alliance of Students v. Matthews, 544 F. 2 d I63 (4th Circ. 1976), 239n

Gay Law Students Association v. Pacific Telephone and Telegraph, 595 P. 2d 592 (Sup. Ct. Cal. I 979), I 9n, 23 In, 249

Gay Lib v. University of Missouri, 4 I 6 F. Supp. 1350 (W. D. Mo. 1976), 558 F. 2d 4 I 3 (8th Circ. I977), I 9n, 239-42

See also Ratchford v. Gay Lib

Gaylord v. Tacoma School District No. ro, 525 P. 2d 804, 559 P. 2d I 340 (Sup. Ct. Wash. I975, I 977), cert. den. 434 U.S. 879 (1977), I9n, 24750

Gay Student Services v. Texas A \& M University, 6I 2 F. 2d I60 (5th Circ. 1980), 239n

Gay Students Organization v. Bonner, 367 F. Supp. 1088 (D. N.H. I 974), 509 F. 2d 652 (Ist Circ. 1974), 238-39, 251

General Electric v. Gilbert, 429 U.S. I 25 (1976), 282n

Gideon v. Wainwright, 372 U.S. 335 (1963), 27n

Gish v. Board of Education, 366 A. 2d I 337 (N.J. Super. Ct. I976), cert. den. 434 U.S. 879 (1977), 247-49, 278

Gitlow v. New York, 268 U.S. 652 (I925), 267n

Glaser v. Marietta, 35 I F. Supp. 555 (W.D. Pa. 1972), I77n

Gonyaw v. Gray, 36 I F. Supp. 366 (D. Vt. 1973), 177n

Goss v. Lopez, 419 U.S. 565 (1975), I9n, I67n, I74n, 175-79, 185, 202

Graham v. Richardson, 403 U.S. 365 (1971), 26n, I I9-20, I 29

Gregg v. Georgia, 428 U.S. I 53 (1976), $178 \mathrm{n}$

Griffin v. Illinois, 35 I U.S. I 2 (1956), $27 \mathrm{n}$

Griggs v. Duke Power Co., 40I U.S. 424 (I97I), 2 I on

Griswold v. Connecticut, 38 I U.S. 479 (1965), I 9n, 21, 230n, 233-37, 25 I

Gurmankin v. Costanzo, 4 I I F. Supp. 982 (E.D. Pa. I 976), 556 F. 2 d I 84

(3d Circ. 1977), 2 I 2-1 3, 27 I

H. L. v. Matheson, 450 U.S. 398, I01 S.Ct. I 169 (1981), I9n, I67n

Hairston v. Drosick, 423 F. Supp. I 80 (S.D. W.Va. I 976), 200n, 205-8

Halderman v. Pennhurst State School and Hospital, 446 F. Supp. 295 (E.D.

Pa. I977), 6 I 2 F. 2d 84 (3d Circ. 1979)

See Pennhurst State School and Hospital v. Halderman

Harris v. McRae, 448 U.S. 297, I00 S.Ct. 267 I (I980), I 27

Healy v. James, 408 U.S. I69 (1972), 237-42, 245

Heart of Atlanta Motel, Inc. v. United States, 379 U.S. 42 I (I964), 33n

Hernandez v. Texas, 347 U.S. 475 (I954), 26n, I42, 271

Hirabayashi v. United States, 320 U.S. 8I (I943), I I 2, I 49, 263

Hobson v. Hansen, 269 F. Supp. 40I (D. D.C. 1967), 203 


\section{Index of Cases}

Hofbauer, matter of, 393 N.E. 2 d 1009 (N.Y. Ct. App. 1979), I9n, 168-72, I $86,188,198,270,280$

Holden v. Hardy, 169 U.S. 366 (1898), 23n

Hoyt v. Florida, 368 U.S. 57 (I961), I 23n

Ingraham v. Wright, 498 F. 2d 248, 525 F. 2d 909 (5th Circ. 1974, 1976), 430 U.S. 65 I (1977), I nn, I67n, I74n, I76-79, 185-86, 246, 266, 270,280

Institutionalized Juveniles v. Secretary of Public Welfare of Pennsylvania, 459 F. Supp. 30 (E.D. Pa. 1978)

See Parham v. J. R. et al.

Irvine v. California, 347 U.S. I 38 (1954), 178

J. L. [sic] v. Parham, 4 I2 F. Supp. I 2 (M.D. Ga. 1976)

See Parham v. J. R. et al.

Jackson v. Bishop, 404 F. 2d 57I (8th Circ. I968), I78n

James v. Wallace, 406 F. Supp. 318 (M.D. Ala. 1976), 203

Jones v. Alfred H. Mayer Corp., 392 U.S. 409 (1968), I4In

Kahn v. Shevin, 416 U.S. 35 I (1974), 28n, 145-46, 27 I

Katzenbach v. McClung, 379 U.S. 904 (I964), 33n

Keyishian v. Board of Regents, 385 U.S. 589 (1967), 244n

Kochman v. Keansburg Board of Education, 305 A. 2 d 807 (N.J. Super. Ct. 1973), I 9n, 247-48

Korematsu v. United States, 323 U.S. 214 (1944), I I 2-14, I 29, I49, 26364

Kremens v. Bartley, 43 I U.S. I 19 (1977), I66n

Kruse v. Campbell, 43 I F. Supp. 180 (E.D. Va. 1977), 204n

Larry P. v. Riles, 343 F. Supp. 1306 (N.D. Cal. 1972), 502 F. 2 d 963 (9th Circ. 1974), 20on

Lochner v. New York, 198 U.S. 45 (1905), i 2n, I05n, 258-59, 278

Lopez v. Williams, 372 F. Supp. 1279 (S.D. Ohio 1973) See Goss v. Lopez

Lora v. Board of Education, 456 F. Supp. 12 II (E.D. N.Y. 1978 ), 623 F. $2 \mathrm{~d}$ 248 (2d Circ. 1980), 200n

Loving v. Virginia, 388 U.S. I (1967), 22n, I I 5-1 8, I25, I 38, 259

McAuliffe v. Mayor of New Bedford, 29 N.E. 517 (Mass. Sup. Jud. Ct. 1892), 244n

McConnell v. Anderson, 3 I6 F. Supp. 809 (D. Minn. 1970), 45 I F. 2 d 193 (8th Circ. 197I), cert. den. 405 U.S. 1046 (1972), 244, 248

McDonald v. Santa Fe Trail Transportation Co., 427 U.S. 273 (1976), I 32 , I35, I49-5I, 27I, 279-80

McKeiver v. Pennsylvania, 403 U.S. 528 (197I), I 9n, I8I-84

McLaughlin v. Florida, 379 U.S. I 84 (1964), I I 5-I 8, I 38 , 259

McLaurin v. Oklahoma State Regents, 334 U.S. 637 (I950), I I 2 n

Massachusetts Board of Retirement v. Murgia, 427 U.S. 307 (1976), 19n, I 27-30, I 54, I 58-61, I 85-86, 263, 266-67, 273 
Matlovich v. Secretary of the Air Force, 414 F. Supp. 690 (D. D.C. 1976), 591 F. 2d 852 (D.C. Circ. 1978), 243

Meyer v. Nebraska, 262 U.S. 390 (1923), I67, I72n, 198

Michael M. v. Superior Court of Sonoma County, 450 U.S. 464, I0I S.Ct. I 200 (I 98I), I 24-25

Mills v. Board of Education, 348 F. Supp. 866 (D. D.C. I972), I 8n, 20I-8

Minersville School District v. Gobitis, 3 Io U.S. 586 (I940), 282n

Mississippi Gay Alliance v. Goudelock, 536 F. 2d 1073 (5th Circ. 1976), cert. den. 430 U.S. 982 (1977), 239n

Missouri ex rel. Gaines v. Canada, 305 U.S. 337 (I938), i I $2 \mathrm{n}$

Morrison v. Board of Education, 46I P. 2 d 375 (Cal. Sup. Ct. 1969), 24344

Muller v. Oregon, 208 U.S. 4 I 2 (I908), 33-34, I 2 I, I 23n

Murgia v. Commonwealth of Massachusetts Board of Retirement, $376 \mathrm{~F}$. Supp. 753 (D. Mass. I 974), 502 F. 2d I I 58 (Ist Circ. 1974)

See Massachusetts Board of Retirement v. Murgia

NAACP v. Alabama, 357 U.S. 449 (I958), 23 in, 238

Naim v. Naim, 350 U.S. 891, 985 (I954), I I $5 \mathrm{n}$

National League of Cities v. Usery, 426 U.S. 833 (1976), 282n

Norton v. Macy, 4I 7 F. 2d I I6I (D.C. Circ. I969), 243n

O’Connor v. Donaldson, 422 U.S. 563 (1975), 20n, 164-65, I 84

Orr v. Orr, 440 U.S. 68 (1979), I46-47, I 50

Oyama v. California, 332 U.S. 63 I (1948), 26n

Pace v. Alabama, I06 U.S. 583 (1883), I09-10, I I 2, I I 5

Palko v. Connecticut, 302 U.S. 319 (1937), 23n

Parham v. J. R. et al., 442 U.S. 584, 99 S.Ct. 2493 (I979), I9n, I64-72, I 84-86, 200-20I, 266, 270, 275

Pennhurst State School and Hospital v. Halderman, 45 I U.S. I, Ior S.Ct. I 53 I (I98I), 219-24

Pennsylvania Association for Retarded Children (PARC) v. Pennsylvania, 334 F. Supp. I 257, 343 F. Supp. 279 (E.D. Pa. I971, 1972), I8n, 20I8

Phillip B., in re, I 56 Cal. Rptr. 48 (Cal. Ct. App. I979), I9n, I68-72, I88, I $98-99,270$

Pickering v. Board of Education, 391 U.S. 563 (I968), 245

Pierce v. Society of Sisters, 268 U.S. 510 (1925), I66-67, I72n, 198

Planned Parenthood Association of Central Missouri v. Danforth, 428 U.S. 52 (1976), I67n

Plessy v. Ferguson, I63 U.S. 537 (1896), I06, I09-1 5, 130, 198, 223, 260

Plyler v. Doe, 50 U.S.L.W. 4650 (1982), 26n, 282n

Prigg v. Pennsylvania, I 6 Peters 539 (I842), 67-70, 73, IOI

Railway Express Agency v. New York, 336 U.S. 106 (1949), 24n

Ratchford v. Gay Lib, 434 U.S. Io80 (1978), I9n, 24I-42, 249

See also Gay Lib v. University of Missouri

Reed v. Reed, 404 U.S. 7I (I97I), 34, I 2 I-22

Regents of the University of California v. Bakke, 438 U.S. 265 (1978), I8n, 


\section{Index of Cases}

29n, I I 6, I I 9n, I 22-26, I 32-33, I 35, I 40-46, I 85-88, 252, 262$63,267-68,270-71$

Reynolds v. Sims, 377 U.S. 533 (1964), I 2n, 282n

Roberts v. Boston, 5 Cushing 198 (Suffolk Co. I849), 77

Roe v. Wade, 410 U.S. I I 3 (1973), i 9n, 230n, 234-37, 276-77

Rostker v. Goldberg, 453 U.S. 57, I0 I S.Ct. 2646 (I98I), I 24-25, I 59

Rowley v. Board of Education, 483 F. Supp. 528 (S.D. N.Y. 1980), 632 F. 2d 947 (2d Circ. 1980), 22 I-22

See also Board of Education v. Rowley

Saal v. Middendorf, 427 F. Supp. 192 (N.D. Cal. 1977), 243n

Sail'er Inn, Inc. v. Kirby, 485 P. 2 d 529 (Cal. Sup. Ct. I97I), 26n

San Antonio Independent School District v. Rodriguez, 4 I I U.S. I (I973), 27n, 28n, I I n , I 20-30, I 5 I, I 84-88, I99, 202-4, 21 5-I6, 25 I-52, 263-64, 267-68, 274, 278

Santosky v. Kramer, 455 U.S. 745 , I02 S.Ct. I 388 (I982), I9n

Schenck v. United States, 249 U.S. 47 (1919), 245, 250, 276-77

Schlesinger v. Ballard, 4 I 9 U.S. 498 (I975), I 45-47

Schware v. Board of Bar Examiners, 353 U.S. 232 (1957), 244n

Scott v. Macy, 349 F. 2d I82, 402 F. 2d 644 (D.C. Circ. 1965, 1968), 243n

Secretary of Public Welfare of Pennsylvania v. Institutionalized Juveniles, 442 U.S. 640, 99 S.Ct. 2523 (I979)

See Parham v. J. R. et al.

Shapiro v. Thompson, 394 U.S. 6 I 8 (1968), 208n

Shelley v. Kraemer, 334 U.S. I (1948), 80

Shelton v. Tucker, 364 U.S. 479 (I960), 238

Sherbert v. Verner, 374 U.S. 398 (1963), 26n

Sims v. Board of Education, 329 F. Supp. 678 (D. N.M. 1971), I77n

Singer v. United States Civil Service Commission, 530 F. 2d 247 (9th Circ. I 976 ), 244n

Sipuel v. Board of Regents, 332 U.S. 63 I (1948), I I $2 \mathrm{n}$

Skinner v. Oklahoma, 3 I 6 U.S. 535 (I942), I I 2 n

Slaughter-House Cases, 83 U.S. 394 (1 873), 106-7, 109, 223, 259

Snowden v. Birmingham-Jefferson County Transit Authority, 407 F. Supp. 394 (N.D. Ala. 1975), I 99n, 209-10

Snyder v. Massachusetts, 29I U.S. 97 (I934), 23n

Southeastern Community College v. Davis, 442 U.S. 397, 99 S.Ct. 236 I

(1979), I 8n, 209n, 213, 216-19, 27 I, 273, 278

See also Davis v. Southeastern Community College

Stanton v. Stanton, 42 I U.S. 7 (I975), I 59n

State v. Yoder, I 82 N.W. 2d 539 (Wis. Sup. Ct. I97I)

See Wisconsin v. Yoder

Steelworkers v. Weber, 443 U.S. I 93 (1979), I 8n, I33-34

Strauder v. West Virginia, Ioo U.S. 664 (I 880), I07-I4, I 4 I

Swann v. Charlotte-Mecklenburg Board of Education, 402 U.S. I (I97I), I $32 \mathrm{n}$

Sweatt v. Painter, 334 U.S. 629 (I950), I I 2 n

Takahishi v. Fish and Game Commission, 334 U.S. 4 IO (I948), 26n

Tate v. Board of Education, 453 F. 2d 975 (8th Circ. 1972), 174n, 175n 


\section{Index of Cases}

Taylor v. Maryland School for the Blind, 408 F. Supp. I48 (D. Md. I976), 205-8

Terminiello v. Chicago, 337 U.S. I (1949), 173-74, I 84

Terry, in re, 265 A. 2 d 350 (Pa. Sup. Ct. 1970) See McKeiver v. Pennsylvania

Tinker v. Des Moines Independent Community School District, 383 F. 2d 988 (8th Circ. 1967), 393 U.S. 503 (1969), 27n, I63, I73-76, I84-86, 230, 250, 271, 280

Trimble v. Gordon, 430 U.S. 762 (1977), 21 , 224n

Twining v. New Jersey, 2 I I U.S. 78 (1908), 178

United Handicapped Federation v. André, 409 F. Supp. 297 (D. Minn. 1976), 558 F. 2d 4 I 3 (8th Circ. 1977), I 99n, 209-10

United States v. Carolene Products, 323 U.S. I44 (I938), 29n, I 20, I85-88, 199, 262-64, 267-68

United States v. Kras, 409 U.S. 434 (1973), $27 n$

United States v. St. Clair, 291 F. Supp. 122 (S.D. N.Y. I968), I 23 n

Vail v. Board of Education, 354 F. Supp. 592 (D. N.H. I973), I74n

Vance v. Bradley, 400 U.S. 93 (I979), I 9n, I 28-30, I 54, I 58-61, I85-86, 262, 267, 273

Virginia, ex parte, 100 U.S. 667 (1 880), 107-8

Vorchheimer v. School District of Philadelphia, 535 F. 2d 880 (3d Circ. I976), 27In

W. v. Family Court, 247 N.E. 2 d 253 (N.Y. Ct. App. I969), I 80 n

See also Winship, in re

Ware v. Estes, 328 F. Supp. 157 (N.D. Tex. 1971), I77n

Weinberger v. Wiesenfeld, 420 U.S. 636 (1975), 144

West Virginia State Board of Education v. Barnette, 3 I 9 U.S. 624 (I943), $282 n$

Wieman v. Updegraff, 344 U.S. I 83 (1952), 244n

Williams v. McNair, 3 I 6 F. Supp. I 34 (D. S.C. I970), 27 In

Winship, in re, 397 U.S. 358 (1970), I8 I-83

See also W. v. Family Court

Wisconsin v. Constantineau, 400 U.S. 433 (1971), 202-3

Wisconsin v. Yoder, 406 U.S. 205 (1972), 163-64, 184-85, 198

Wolf v. Colorado, 338 U.S. 25 (1949), 178

Wood v. Davison, 35 I F. Supp. 543 (N.D. Ga. 1972), 238

Wyatt v. Stickney, 325 F. Supp. 78I, 334 F. Supp. I 34, 334 F. Supp. 373

(M.D. Ala. 1971, 1972), 203, 219-20

Yates v. United States, 355 U.S. 66 (1957), 240n

Yick Wo v. Hopkins, I I 8 U.S. 356 (1886), 109, I I 3, 259-60 